\title{
Patchy alopecia areata sparing gray hairs: a case series
}

\author{
Wei-Xue Jia, Qiu-Xia Mao, Xue-Min Xiao, Zhi-Liang Li, Rui-Xing Yu, Cheng-Rang Li
}

Institute of Dermatology, Chinese Academy of Medical Sciences and Peking Union Medical College, Nanjing, China Head of Institute: Prof. Bao-Xi Wang

Postep Derm Alergol 2014; XXXI, 2: 113-116

DOI: $10.5114 /$ pdia.2014.40956

\begin{abstract}
Alopecia areata is an unpredictable, non-scarring hair loss condition. Patchy alopecia areata sparing gray hairs is rare. Here we present 4 cases with patchy non-scarring hair loss, which attacked pigmented hairs only and spared gray hairs. It should be differentiated from vitiligo, colocalization of vitiligo and alopecia areata, and depigmented hair regrowth after alopecia areata.
\end{abstract}

Key words: alopecia areata, gray hairs, pigmented hairs.

\section{Introduction}

Alopecia areata is an unpredictable, usually patchy, non-scarring hair loss condition. The exact pathogenesis of the disorder remains to be clarified. It is mainly considered a T-cell-mediated autoimmune disease [1]. Alopecia areata is manifested as round or oval, well-demarcated patches, most commonly on the scalp and in the region of the beard. Patchy alopecia areata sparing gray hairs is rare.

Here we present 4 cases with patchy alopecia areata sparing gray hairs to bring attention to this unusual phenomenon and possible pathogenic mechanisms.

\section{Case reports}

Case 1

A 53-year-old man presented with patches of hair loss sparing non-pigmented hairs on the occipital scalp, which had steadily worsened over a period of 2 weeks. Prior to his alopecia, he had a mixture of dark and white hairs. He denied any other relevant or serious illness histories. On physical examination, two patches of well-demarcated lesions of about $10 \mathrm{~cm} \times 5 \mathrm{~cm}$ and $3 \mathrm{~cm} \times 2 \mathrm{~cm}$ on the occipital scalp were seen. Gray hairs were distributed among the patch of hair loss, with similar density to the gray hairs over normal area of scalp and similar diameter and length to the pigmented hairs. No pigmented hairs were on the shedding patches (Figure 1). Results of laboratory examinations, including the blood routine test, C-reactive protein (CRP), erythrocyte sedimentation rate (ESR), IG, complement and thyroid function tests, were within the normal range. Alopecia areata was diagnosed.

\section{Case 2}

A 50-year-old man presented with a patch of hair loss on the occipital scalp for one month which attacked pigmented hairs only. Prior to his alopecia, he had black hair with some gray. He denied any medical problems. On physical examination, a round well-demarcated patch of hair loss with a diameter of about $1.5 \mathrm{~cm}$ was seen on the occipital scalp, among which some gray hairs were distributed. The density and length of gray hairs on the patch of hair loss were similar to those on the normal area of the scalp. No pigmented hairs were seen over the hair loss patch (Figure 2). Alopecia areata was diagnosed.

\section{Case 3}

A 41-year-old man presented with a patch of hair loss for 2 months. Prior to his alopecia, he had black hair which were streaked with gray. On physical examination, a well-defined patch of hair loss presented on the scalp with a diameter of about $4 \mathrm{~cm}$ was seen. Some gray hairs were randomly distributed among the balding patch, which showed the similar length with neighboring pigmented hairs. A few pigmented hairs were seen over the patch of hair loss (Figure 3). Alopecia areata was diagnosed.

\section{Case 4}

A 38-year-old man presented with a patch of hair loss on the vertex which attacked pigmented hair only and spared gray hairs. He reported that two small patches of hair loss on the vertex started 3 weeks before presentation, which had been gradually enlarging and joining together. Prior to his alopecia, he had black hair with

Address for correspondence: Cheng-Rang Li, Institute of Dermatology, Chinese Academy of Medical Sciences and Peking Union Medical College, No. 12, Jiangwangmiao Street, Nanjing, China, e-mail: nylcr72@163.com Received: 8.11.2013, accepted: 27.11.2013. 


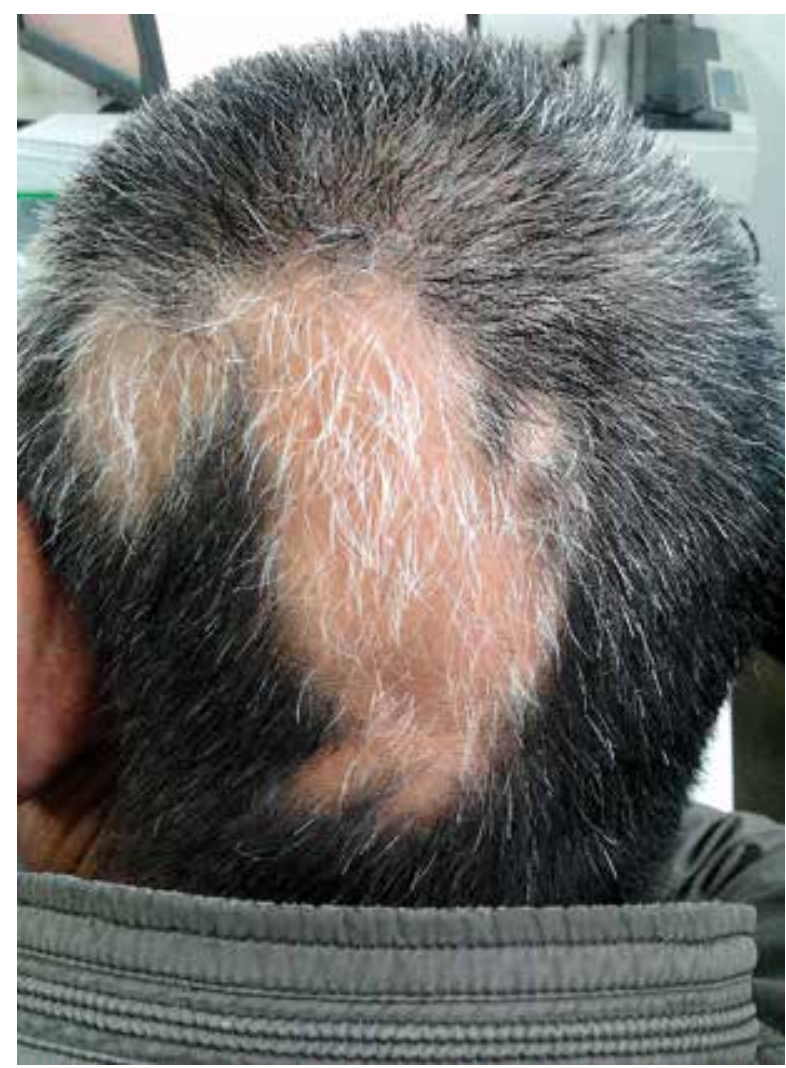

Figure 1. Case 1. Two patches of well-demarcated lesions of about $10 \mathrm{~cm} \times 5 \mathrm{~cm}$ and $3 \mathrm{~cm} \times 2 \mathrm{~cm}$ on the occipital scalp. Gray hairs were distributed among the patch of hair loss, with similar density to the gray hairs over normal area of scalp and similar diameter and length to the pigmented hairs. No pigmented hairs were on the shedding patches

some gray. On physical examination, there was a patch of well-demarcated hair loss lesion with a diameter of about $12 \mathrm{~cm} \times 7 \mathrm{~cm}$ on the vertex of the scalp. Some gray hairs and black hairs were distributed among the patch of hair loss, which showed the similar diameter and length to the hair on the normal scalp. Exclamation mark hairs were seen at the periphery of areas of hair loss (Figure 4). Alopecia areata was diagnosed.

\section{Discussion}

Alopecia areata pathogenesis is not fully understood. It is hypothesized to be an organ-specific autoimmune disease mediated by $T$ lymphocytes against hair follicles. There are other proposed origins reported, including genetic constitution, cytokines, infectious agents, emotional stress and so on [2]. Alopecia areata typically presents with round patches of hair loss. The patches may be single or multiple. Other presentations include alopecia totalis, alopecia universalis, ophiasis and diffuse variant of alopecia areata. Any hair-bearing area can be affected. A positive pull test with telogen or dystrophic anagen hairs at the margins of the lesion suggests ac-

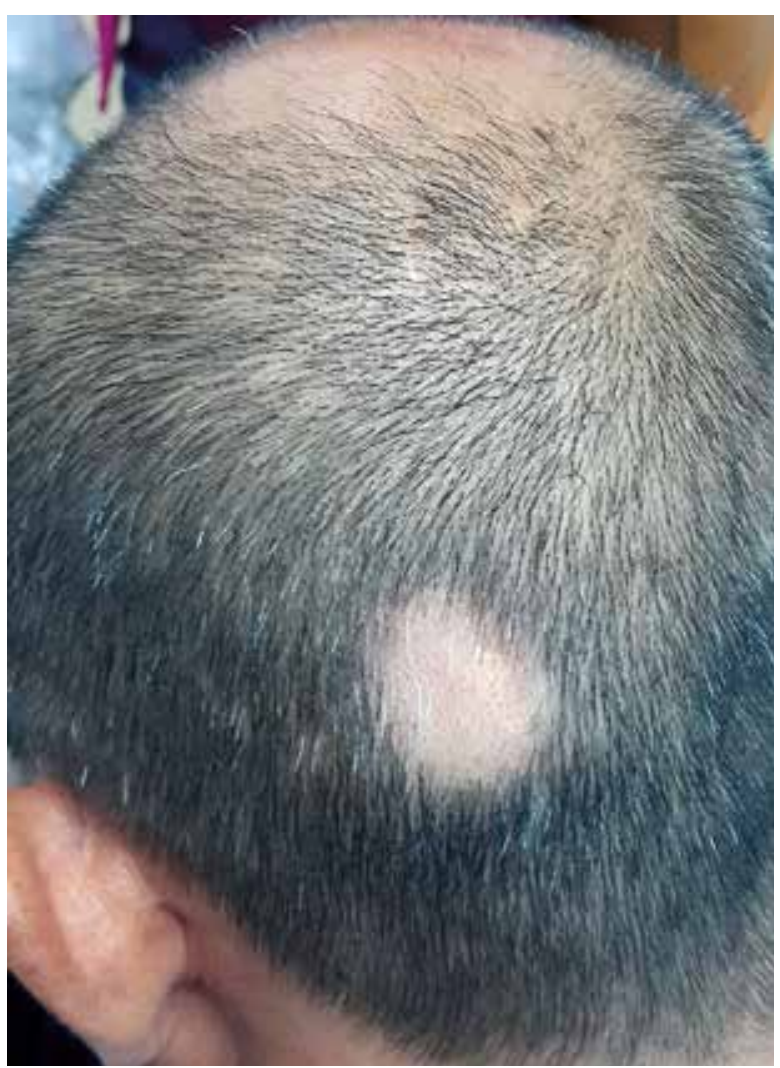

Figure 2. Case 2. A patch of well-demarcated, about $1.5 \mathrm{~cm}$ sized patchy hair loss on the occipital scalp, among which some gray hairs were distributed. No pigmented hairs were seen over the hair loss patch

tivity. Exclamation mark hairs are another sign of acute disease [3]. The presence of alopecia areata is associated with a higher frequency of atopy, autoimmune thyroid disease and vitiligo.

The four cases described here presented with patchy non-scarring hair loss which attacked pigmented hairs only and spared gray hairs. All these characteristics led to the diagnosis of patchy alopecia areata sparing gray hairs. A similar case has been reported, and they believe this feature may add a new subtype of alopecia areata [4]. Non-pigmented hairs may initially be spared, and, as a result, some patients with diffuse involvement note rapid whitening of the hair. The case of sudden whitening of the hair was first reported in the Talmud [5]. And a few cases have been reported afterwards [6-8].

The appearance of hair whitening in both localized and diffuse forms of alopecia areata is thought to be attributable to that pigmented hairs are preferentially lost. Currently available evidence suggests that alopecia areata can be considered a T-cell-mediated autoimmune disease in which the gradual loss of protection provided by immune privilege of the normal hair follicle plays 


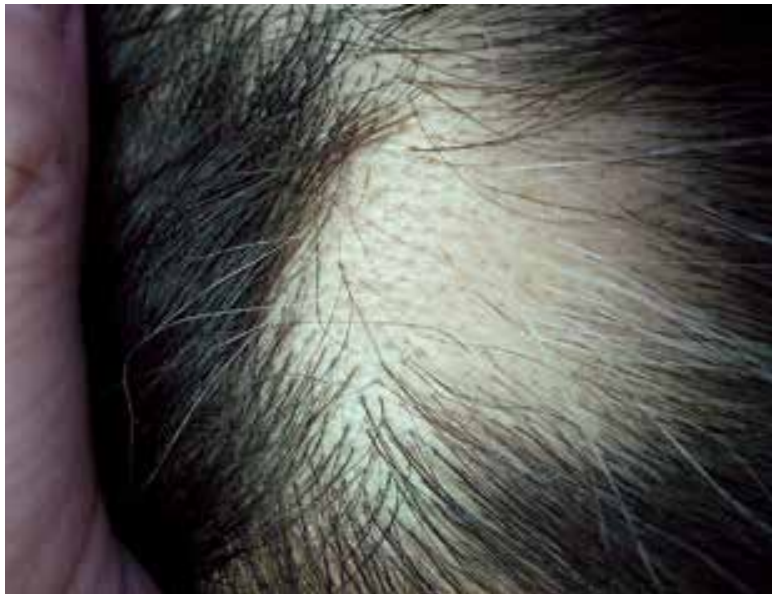

Figure 3. Case 3. A round, well-defined patch of hair loss on the scalp with a diameter of about $4 \mathrm{~cm}$. Some gray hairs were randomly distributed among the balding patch. A few pigmented hairs were seen over the patch of hair loss

an important role [9]. It is demonstrated that hair follicle melanocyte is one of the targets [10]. Ultrastructural evidence proves that hair bulb melanocytes are specifically damaged in acute alopecia areata, even before any damage is morphologically detectable in hair bulb keratinocytes [11]. Epitope spreading is likely to have a role in the broad nature of the anti-melanocyte response [12].

Patchy alopecia areata sparing gray hairs should be differentiated from vitiligo, colocalization of vitiligo associated with alopecia areata, and depigmented hair regrowth after alopecia areata. Vitiligo of the scalp usually presents as a localized patch of white, commonly with total depigmentation of hair or only a few follicles on amelanotic macule may be involved. The scalp of the patients in our paper did not present with the white patch, and there had been no change of the color of lesional hairs. The density of black hair in the lesion reduced while that of gray hair did not. Therefore, vitiligo and colocalization of vitiligo associated with alopecia areata can be ruled out. The number of melanocytes and their melanization in regrowing white anagen hair follicles of alopecia areata were much less than in the normal pigmented follicle $[2,13]$. It is also recognized that regrowth of hair with a patch of alopecia areata is often depigmented, which could be temporary or persistent [14]. The cases described here had a short course, and the gray hairs in the patch of hair loss showed a similar diameter and length to the hair on normal scalp. Accordingly, depigmented hair regrowth after alopecia areata can be ruled out.

A wide range of treatments is available for the management of alopecia areata. The treatment may include intralesional corticosteroids, topical corticosteroids, minoxidil, anthralin, or immunotherapy, systemic corticosteroids, photochemotherapy, and combination ther-

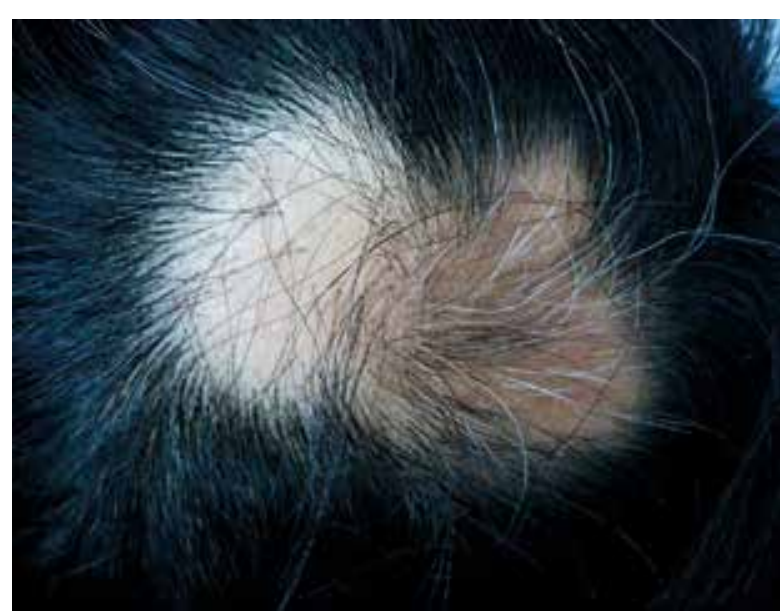

Figure 4. Case 4. A patch of well-demarcated hair loss lesion with a diameter of about $12 \mathrm{~cm} \times 7 \mathrm{~cm}$ on the vertex of the scalp. Some gray hairs and black hairs were distributed among the patch of hair loss. Exclamation mark hairs at the periphery of areas of hair loss

apy [15]. Children with severe and long-lasting alopecia areata have the most difficult prognosis. A positive family history, nail involvement, atopy, and associated changes are negative prognostic factors [3]. All the cases described here had a short course, and the longest course was only 2 months. In case 3 and case 4, a small amount of pigmented hairs and some gray hairs were distributed among the patches of hair loss, even though only pigmented hairs were attacked. These two aspects suggest that patchy alopecia areata sparing gray hairs may be forme frusta of alopecia areata, which is a favorable prognostic factor.

\section{Conclusions}

The phenomenon that patchy alopecia areata sparing gray hairs is the inspiration for further investigations into progression and prognosis of alopecia areata, which recommend more case reports and fundamental researches.

\section{Conflict of interest}

All authors declare no conflict of interest.

\section{References}

1. Dawe RS. Alopecia Areata. N Engl I Med 2012; 367: 279-80.

2. Madani S, Shapiro J. Alopecia areata update. J Am Acad Dermatol 2000; 42: 549-66.

3. Finner AM. Alopecia areata: clinical presentation, diagnosis, and unusual cases. Dermatol Ther 2011; 24: 348-54.

4. Tan C, Zhu WY, Min ZS. A case of patchy alopecia areata sparing lesional greying hairs. Int I Dermatol 2008; 47: 864-5.

5. Goldenhersh MA. Rapid whitening of the hair first reported in the Talmud. Possible mechanisms of this intriguing phenomenon. Am J Dermatopathol 1992; 14: 367-8. 
6. Plinck EP, Peereboom-Wynia JD, Vuzevski VD, et al. Turning white overnight: is it possible? Ned Tijdschr Geneeskd 1993; 137: 1207-10.

7. Dinh QQ, Chong AH. A case of widespread non-pigmented hair regrowth in diffuse alopecia areata. Australas J Dermatol 2007; 48: 221-3.

8. Tan SP, Weller RB. Sudden whitening of the hair in an 82-year-old woman: the 'overnight greying' phenomenon. Clin Exp Dermatol 2012; 37: 458-9.

9. Gilhar A, Etzioni A, Paus R. Alopecia areata. N Engl J Med 2012; 366: 1515-25.

10. Nagai H, Oniki S, Oka M, et al. Induction of cellular immunity against hair follicle melanocyte causes alopecia. Arch Dermatol Res 2006; 298: 131-4.

11. Tobin DJ, Fenton DA, Kendall MD. Ultrastructural observations on the hair bulb melanocytes and melanosomes in acute alopecia areata. J Invest Dermatol 1990; 94: 803-7.

12. Gilhar A, Landau M, Assy B, et al. Melanocyte-associated T cell epitopes can function as autoantigens for transfer of alopecia areata to human scalp explants on Prkdc(scid) mice. J Invest Dermatol 2001; 117: 1357-62.

13. Messenger AG, Bleehen SS. Alopecia areata: light and electron microscopic pathology of the regrowing white hair. $\mathrm{Br}$ J Dermatol 1984; 110: 155-62.

14. Wade MS, Sinclair RD. Persistent depigmented regrowth after alopecia areata. J Am Acad Dermatol 2002; 46: 619-20.

15. Gutfreund K, Bienias W, Szewczyk A, Kaszuba A. Topical calcineurin inhibitors in dermatology. Part I: properties, method and effectiveness of drug use. Postep Derm Alergol 2013; 30: 165-9. 